what genes regulated by PPAR $\gamma$ are antagonistic to osteoblast differentiation?

\section{PPAR $\gamma$ and osteoporosis: from bench to clinic}

Agents currently approved for treatment of osteoporosis act largely by inhibiting bone resorption. These include hormone replacement therapy, calcium and vitamin D supplementation, and bisphosphonatebased drugs (alendronate sodium/Fosamax and risedronate/Actonel) (11). The only exception is the recently approved parathyroid hormone (PTH)-derived peptide Forteo, which can stimulate bone formation. However, significant disadvantages exist for PTH treatment. For example, sustained exposure to elevated PTH levels results in net bone loss, so intermittent exposure by daily injection is necessary (12). New medicines that promote bone formation/osteoblastogenesis with fewer side effects could have great utility in the treatment of osteoporosis.

The findings of Akune et al. suggest that aspects of the PPAR $\gamma$ pathway might be amenable to pharmacologic intervention in osteoporosis (10). One possibility raised by the authors is the use of PPAR $\gamma$ modulators or antagonists. Some support for this idea comes from a recent study that identified $12 / 15$-lipoxygenase as a susceptibility gene for bone mineral density in mice (13). The authors of this study hypothesized that
PPAR $\gamma$ may be involved in these effects, since 12/15-lipoxygenase is capable of generating PPAR $\gamma$ ligands from linoleic/arachidonic acids and oxidized LDL $(14,15)$. However, the use of PPAR $\gamma$ modulators/antagonists for osteoporosis needs to be approached with caution given the critical role of PPAR $\gamma$ in mammalian physiology. Thiazolidinediones, a class of synthetic PPAR $\gamma$ agonists, are currently used to treat type 2 diabetes. The possibility that an antagonist to PPAR $\gamma$ might exacerbate insulin resistance, particularly in susceptible individuals, needs to be carefully considered. In the case of the estrogen receptor, it has been possible to identify compounds that have tissue-specific actions on a nuclear receptor. A bone-selective PPAR $\gamma$ modulator, in this case an antagonist, may be required to exploit PPAR $\gamma$ as a target in osteoporosis.

Address correspondence to: Peter Tontonoz, Howard Hughes Medical Institute, Department of Pathology and Laboratory Medicine, UCLA, Box 951662, Los Angeles, California 90095-1662, USA. Phone: (310) 206-4546; Fax: (310) 267-0382; E-mail: ptontonoz@mednet.ucla.edu.

1. Karsenty, G., and Wagner, E.F. 2002. Reaching a genetic and molecular understanding of skeletal development. Dev. Cell. 2:389-406.

2. Boyle, W.J., Simonet, W.S., and Lacey, D.L. 2003. Osteoclast differentiation and activation. Nature. 423:337-342.
3. Harada, S., and Rodan, G.A. 2003. Control of osteoblast function and regulation of bone mass. Nature. 423:349-355.

4. Rosen, E.D., and Spiegelman, B.M. 2000. Molecular regulation of adipogenesis. Annu. Rev. Cell Dev. Biol. 16:145-171.

5. Hu, E., Tontonoz, P., and Spiegelman, B.M. 1995. Transdifferentiation of myoblasts by the adipogenic transcription factors PPAR gamma and C/EBP alpha. Proc. Natl. Acad. Sci. U. S. A. 92:9856-9860.

6. Skillington, J., Choy, L., and Derynck, R. 2002. Bone morphogenetic protein and retinoic acid signaling cooperate to induce osteoblast differentiation of preadipocytes. J. Cell Biol. 159:135-146.

7. Jeon, M.J., et al. 2003. Activation of peroxisome proliferator-activated receptor-gamma inhibits the Runx2-mediated transcription of osteocalcin in osteoblasts. J. Biol. Chem. 278:23270-23277.

8. Lecka-Czernik, B., et al. 2002. Divergent effects of selective peroxisome proliferator-activated receptorgamma 2 ligands on adipocyte versus osteoblast differentiation. Endocrinology. 143:2376-2384

9. Meunier, P., Aaron, J., Edouard, C., and Vignon, G. 1971. Osteoporosis and the replacement of cell populations of the marrow by adipose tissue. A quantitative study of 84 iliac bone biopsies. Clin. Orthop. 80:147-154.

10. Akune, T., et al. 2004. PPAR $\gamma$ insufficiency enhances osteogenesis through osteoblast formation from bone marrow progenitors. J. Clin. Invest. 113:846-855. doi:10.1172/JCI200419900.

11. Prestwood, K.M., Pilbeam, C.C., and Raisz, L.G. 1995. Treatment of osteoporosis. Annu. Rev. Med. 46:249-256.

12. Berg, C., Neumeyer, K., and Kirkpatrick, P. 2003. Teriparatide. Nat. Rev. Drug Discov. 2:257-258.

13. Klein, R.F., et al. 2004. Regulation of bone mass in mice by the lipoxygenase gene Alox15. Science. 303:229-232.

14. Huang, J.T., et al. 1999. Interleukin-4-dependent production of PPAR-gamma ligands in macrophages by 12/15-lipoxygenase. Nature. 400:378-382.

15. Tontonoz, P., Nagy, L., Alvarez, J.G., Thomazy, V.A., and Evans, R.M. 1998. PPARgamma promotes monocyte/macrophage differentiation and uptake of oxidized LDL. Cell. 93:241-252.

\title{
Predicting the clinical course of prostate cancer
}

\author{
James McKiernan ${ }^{1}$ and Mitchell C. Benson ${ }^{1,2}$
}

1Department of Urology and ${ }^{2 H e r b e r t ~ I r v i n g ~ C o m p r e h e n s i v e ~ C a n c e r ~ C e n t e r, ~ C o l u m b i a ~ U n i v e r s i t y ~ C o l l e g e ~ o f ~ P h y s i c i a n s ~ a n d ~ S u r g e o n s, ~ N e w ~ Y o r k, ~ N e w ~ Y o r k, ~ U S A . ~}$

\begin{abstract}
Risk stratification in prostate cancer remains a significant clinical challenge. A study in this issue of the JCI describes an exciting application of highthroughput functional genomic technology to further refine our understanding of treatment failure risk in prostate cancer patients (see the related article beginning on page 913).
\end{abstract}

Since Walsh and Donker first described the pelvic anatomy that allowed for the development of the nerve-sparing anatomic radical prostatectomy in 1982 , the mor-

Nonstandard abbreviations used: prostate-specific antigen (PSA).

Conflict of interest: The authors have declared that no conflict of interest exists.

Citation for this article:

J. Clin. Invest. 113:806-808 (2004).

doi:10.1172/JCI200421310. bidity associated with the surgical treatment of clinically localized prostate cancer has decreased substantially (1). The subsequent advent of prostate-specific antigen (PSA) screening has led to a substantial stage migration in newly diagnosed adenocarcinoma of the prostate, and this has resulted in a higher likelihood of surgical cure. Despite these therapeutic advances, our ability to accurately predict the risk of treatment failure for an individual patient with prostate cancer remains limited. The current tools we utilize to guide critical decisions, such as whether or how aggressively to treat prostate cancer, are based on serum PSA levels, biopsy Gleason score, and clinical stage. Despite the incorporation of powerful multifactorial nomograms into our decision process, the ability to predict individual patient outcome remains limited $(2,3)$.

\section{Novel prognostic indicators}

In this issue of the JCI, a report by Glinsky et al. attempts to advance our understanding and ability to stratify the risk of treatment failure for patients with localized prostate cancer undergoing radical 
A

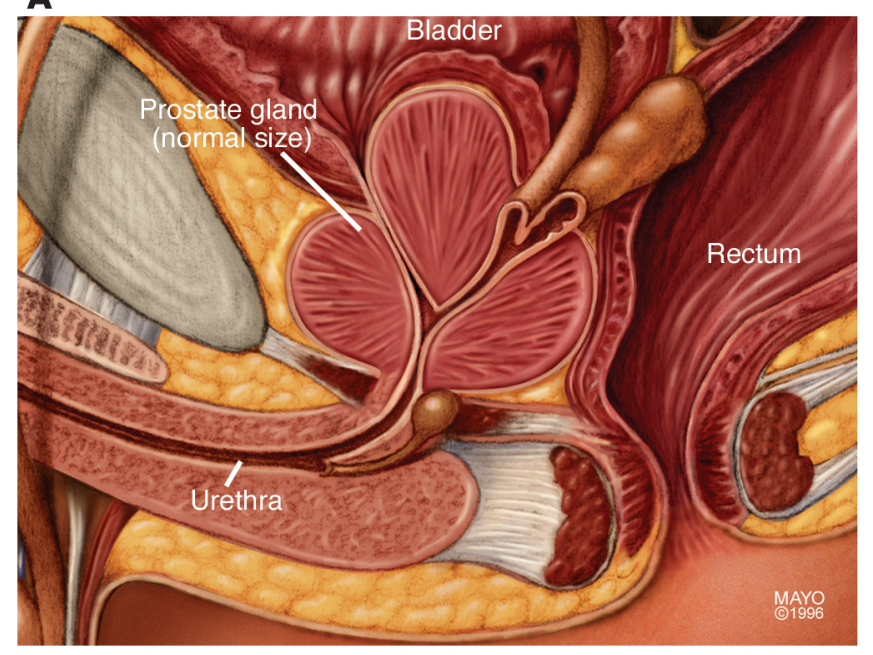

B

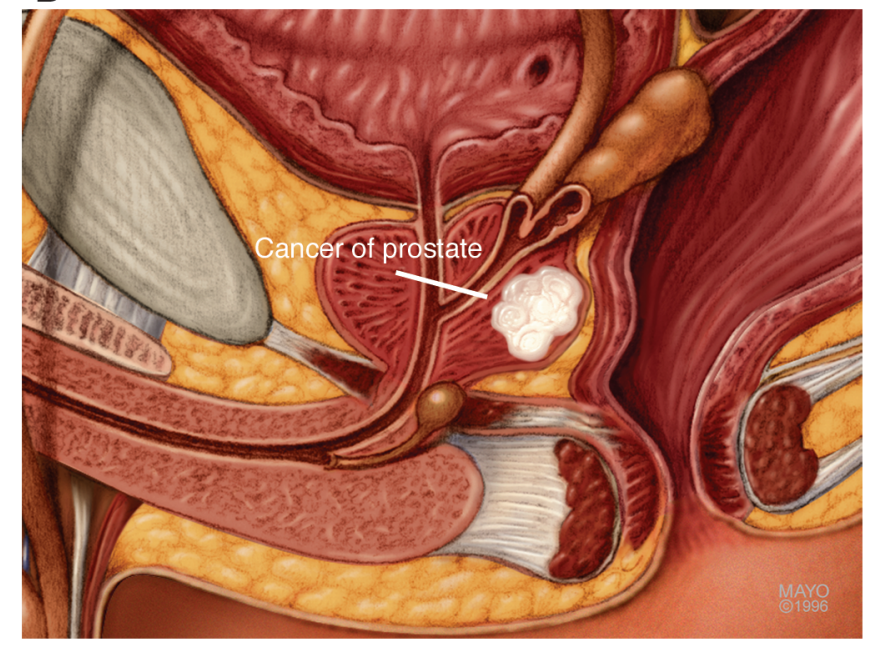

Figure 1

Prostate cancer is the second most common malignancy in men. The prostate gland $(\mathbf{A})$ is located just below the male bladder and can undergo a period of growth beginning in middle age. Malignant tumors of glandular origin (B) are usually adenocarcinomas. Early detection is possible through annual digital rectal examinations and routine PSA testing. Glinksy et al. (4) use gene expression profiling to identify and test prognostic indicators for prostate cancer. The authors developed several genetic "signatures" able to discriminate recurrent versus nonrecurrent disease. Image copyright of Mayo Foundation for Medical Education and Research. All rights reserved. Used with permission from www.MayoClinic.com.

prostatectomy (4). Relying on the enormous power of recent advances in functional human genomics, Glinsky et al. have analyzed the gene expression profiles of human prostate cancer samples in a total of 100 different tumors. The authors screened 21 patients for 12,625 gene transcripts to identify genes that would predict relapse-free survival following radical prostatectomy using the Affymetrix GeneChip system. Subsequently, using a smaller set of highly discriminating fivegene clusters, they were able to predict clinical outcome in a larger validation set of 79 patients.

This study represents the largest clinical series of genomic classification in prostate cancer published to date using a highthroughput functional human genomic technique. The virtually unlimited power of microarray technology to discriminate minute genetic differences in clinical specimens has the potential to revolutionize the power of clinicians to accurately identify high-risk groups of patients (5). However, several potential drawbacks in the current study should be noted. First, this study was performed using the postoperative radical prostatectomy specimen as the tissue source. This is likely due to the greater ease of tissue acquisition in this setting. In order to best assist in clinical decision making, predictive information should be available prior to definitive treatment. This would require DNA analysis of transrectal ultrasound-guided biopsy material. This approach is subject to the unavoidable risk of biopsy sampling error due to the known heterogeneity of multifocal prostate cancer. This heterogeneity is currently manifested by assessments of variability of Gleason Score within a given tumor. It remains to be determined whether distinct foci of cancer within a given tumor will have similar or different genetic profiles. The postoperative risk stratification utilized in the current study is only able to assist in choosing patients in need of early adjuvant therapy or closer follow-up.

\section{Statistical analysis}

In addition, although the authors present a detailed analysis of their data that suggests that these discriminating gene clusters can predict clinical outcome of prostate cancer beyond the traditional factors such as stage, Gleason score, and PSA level, their univariate approach to this analysis is flawed (4). By separating patient groups into high Gleason score or high PSA or high stage and then allowing the cluster analysis to discriminate outcome, they have limited the power of these traditional risk factors, which should be considered simultaneously in each case. A potentially more powerful approach to this problem would be to add gene cluster analysis data in a multivariate model which already includes stage, Gleason score, and PSA level to determine if the predictive ability of gene cluster analysis is in fact additive to current approaches (6). It remains to be determined how accurate and individually predictive such an approach will be. Further stratification of a given patient's risk of treatment failure is important, but the ultimate goal must remain accurate and reproducible individual prediction. Only then will such approaches accomplish more than altering informed consent in selection of therapy.

Despite these limitations the presented data should be heralded as a significant advance in our ability to leverage the awesome power of functional genomics in a clinically useful fashion. It is this translational approach to risk stratification that is most likely to lead to progress in our understanding of each individual prostate cancer patient's risk of treatment failure and allow us to intelligently counsel patients regarding what today remains a complex and often confusing array of treatment options.

Address correspondence to: Mitchell C. Benson, Department of Urology, Irving Pavilion, 11th floor, 161 Fort Washington Avenue, New York, New York 10032, USA. Phone: (212) 305-5201; Fax: (212) 305-6813; E-mail: mcb2@columbia.edu. 
1. Walsh, P.C., and Donker, P.J. 1982. Impotence following radical prostatectomy: insight into etiology and prevention. J. Urol. 128:492-497.

2. Kattan, M.W., Eastham, J.A., Stapleton, A.M., Wheeler, T.M., and Scardino, P.T. 1998. A preoperative nomogram for disease recurrence following radical prostatectomy for prostate cancer. J. Natl. Cancer Inst. 90:766-771.

3. D’Amico, A.V., et al. 1999. Pretreatment nomogram for prostate-specific antigen recurrence after radical prostatectomy or external-beam radiation therapy for clinically localized prostate cancer. J. Clin. Oncol. 17:168-172.

4. Glinsky, G.V., Glinskii, A.B., Stephenson, A.J., Hoffman, R.M., and Gerald, W.L. 2004. Gene expression profiling predicts clinical outcome of prostate cancer. J. Clin. Invest. 113:913-923. doi:10.1172/ JCI200420032.

5. Glinsky, G.V., Ivanova, Y.A., and Glinskii, A.B. 2003.
Malignancy-associated regions of transcriptional activation: gene expression profiling identifies common chromosomal regions of recurrent transcriptional activation in human prostate, breast, ovarian and colon cancers. Neoplasia. 5:221-228.

6. Kattan, M.W., Wheeler, T.M., and Scardino, P.T. 1999. Postoperative nomogram for disease recurrence after radical prostatectomy for prostate cancer. J. Clin. Oncol. 17:1499-1507.

\title{
The immune response to AIDS virus infection: good, bad, or both?
}

\author{
Steven G. Deeks ${ }^{1}$ and Bruce D. Walker ${ }^{2}$
}

\begin{abstract}
${ }^{1}$ Department of Medicine, University of California, San Francisco, San Francisco, California, USA. ${ }^{2}$ Howard Hughes Medical Institute and Partners AIDS Research Center, Massachusetts General Hospital and Division of AIDS, Harvard Medical School, Boston, Massachusetts, USA.
\end{abstract}

\begin{abstract}
A potent antigen-specific $T$ cell response to HIV infection can contribute to the control of viral replication and is therefore beneficial to the host. However, HIV-mediated increases in generalized $\mathrm{T}$ cell activation also appear to accelerate both viral replication and $\mathrm{CD} 4^{+} \mathrm{T}$ cell depletion. A new study in the JCI attempts to experimentally distinguish the beneficial versus harmful aspects of this immune response (see the related article beginning on page 836).
\end{abstract}

Acute HIV infection is associated with symptoms almost everyone has experienced - fever, sore throat, swollen glands, and often a transient rash. These are not specific to HIV, but rather a consequence of the high level of immune activation that is induced in defense of HIV and other acute viral infections. Although a strong immune response is presumably beneficial in most acute infections, specific properties of HIV raise the question as to whether immune activation in this setting may also have harmful consequences. HIV selectively infects and replicates in activated CD4 cells, suggesting that the antiviral effects of the acute immune response may be counterbalanced by the detrimental effects of adding additional fuel (i.e., activated CD4 $\mathrm{T}$ cells) to the fire. The proinflammatory aspects of HIV infection also result in the activation and proliferation of $\mathrm{CD}^{+} \mathrm{T}$ cells specific for antigens other than HIV (the so-called "innocent bystanders"), which can also become productively infected with HIV, albeit at a lower frequency (1). Thus, a stronger immune response to HIV might have the paradoxical effect of

Nonstandard abbreviation used: simian immunodeficiency virus (SIV).

Conflict of interest: The authors have declared that no conflict of interest exists.

Citation for this article:

J. Clin. Invest. 113:808-810 (2004).

doi:10.1172/JCI200421318. enhancing viral replication and accelerating disease progression.

Unfortunately, this synergistic interaction between immune activation and viral replication is only part of the story. More importantly from the host's perspective, chronic heightened activation of the immune system may also contribute in a direct manner to progressive $\mathrm{CD}^{+} \mathrm{T}$ cell depletion. One widely accepted model of HIV immunopathogenesis postulates that heightened immune and proliferation of memory-effector $\mathrm{CD}^{+}$ $T$ cells (2). These cells are destined to die rapidly as a consequence of activationinduced cell death and/or due to direct infection by HIV. Over time, the naive and central memory pools become exhausted and unable to generate new primary responses or maintain the peripheral $\mathrm{CD}^{+}$ $\mathrm{T}$ cell count $(3,4)$.

Given this background, some have questioned whether the immune response to HIV might cause more harm than good. Indeed, substantial circumstantial evidence for such paradoxical effects exists, including the observation that immune activation is a strong independent predictor of disease outcome in antiretroviraluntreated and -treated individuals $(5,6)$. Along these lines, much has recently been made of the observation that sooty mangabeys, the natural host of simian immunodeficiency virus (SIV), support activation results in accelerated activation high levels of viral replication but fail to exhibit a clear increase in immune activation and/or inflammation $(7,8)$. These animals appear to tolerate SIV infection quite well, having life spans that are not dramatically different from those of uninfected animals. In contrast, when SIV is experimentally transferred to rhesus macaques, dramatic increases in immune activation occur, and animals progress rapidly to AIDS and death.

\section{Separating the protective versus destructive aspects of the immune response}

In this issue of the JCI, Garber and colleagues use a clever experimental approach in an attempt to differentiate the potential harmful aspects of virusmediated increases in $\mathrm{T}$ cell activation and/or proliferation from the benefits associated with the generation of an effective SIV-specific T cell response (9). Four adult rhesus macaques were treated with a monoclonal antibody combination designed to block costimulation and thereby eliminate cellular activation during acute SIV infection. CTLA4Ig was used to block CD4 stimulation through CD28CD80/86 interactions, and anti-CD40L was administered to block CD4 stimulation through CD40-CD40L interactions. The effect of this treatment, administered before and during acute SIV infection, was compared to the effect of no treatment in four acutely infected control animals. Viral load, $T$ cell activation, antigen-specific $T$ cell and $B$ cell immune responses, and the emergence of CTL escape mutations were closely monitored during the first few months of infection. 